\title{
Single point mutation in Rabenosyn-5 in a female with intractable seizures and evidence of defective endocytotic trafficking
}

Sylvia Stockler ${ }^{1,2,3^{*}+}$, Silvia Corvera ${ }^{9,10 \dagger}$, David Lambright ${ }^{9,10}$, Kevin Fogarty ${ }^{9,10}$, Ekaterina Nosova ${ }^{3,4,5}$, Deborah Leonard ${ }^{9,10}$, Robert Steinfeld ${ }^{11}$, Cameron Ackerley ${ }^{12}$, Casper Shyr ${ }^{3,4,5}$, Nicolas Au ${ }^{6}$, Kathrin Selby ${ }^{2,7}$, Margot van Allen ${ }^{5}$, Hilary Vallance ${ }^{3,6,8}$, Ron Wevers ${ }^{13}$, David Watkins ${ }^{14}$, David Rosenblatt ${ }^{14}$, Colin J Ross ${ }^{2,3,4}$, Elizabeth Conibear ${ }^{4,5}$, Wyeth Wasserman ${ }^{3,4,5}$ and Clara van Karnebeek ${ }^{1,2,3,4}$

\begin{abstract}
Background: We report a 6.5 year-old female with a homozygous missense mutation in ZFYVE20, encoding Rabenosyn-5 (Rbsn-5), a highly conserved multi-domain protein implicated in receptor-mediated endocytosis. The clinical presentation includes intractable seizures, developmental delay, microcephaly, dysostosis, osteopenia, craniofacial dysmorphism, macrocytosis and megaloblastoid erythropoiesis. Biochemical findings include transient cobalamin deficiency, severe hypertriglyceridemia upon ketogenic diet, microalbuminuria and partial cathepsin D deficiency.

Methods and results: Whole exome sequencing followed by Sanger sequencing confirmed a rare (frequency:0.003987) homozygous missense mutation, g.15,116,371 G > A (c.1273G > A), in ZFYVE20 resulting in an amino acid change from Glycine to Arginine at position 425 of the Rbsn protein (p.Gly $425 \mathrm{Arg}$ ), as the only mutation segregating with disease in the family. Studies in fibroblasts revealed expression and localization of Rbsn-5G425R in wild-type manner, but a 50\% decrease in transferrin accumulation, which is corrected by wild-type allele transfection. Furthermore, the patient's fibroblasts displayed an impaired proliferation rate, cytoskeletal and lysosomal abnormalities.
\end{abstract}

Conclusion: These results are consistent with a functional defect in the early endocytic pathway resulting from mutation in Rbsn-5, which secondarily disrupts multiple cellular functions dependent on endocytosis, leading to a severe multi-organ disorder.

Keywords: Internalization, Receptor endocytosis, Recycling, FYVE domain, Rab GTPase, Epilepsy, Cathepsin D, Vitamin B12, Inborn error of metabolism

\section{Introduction}

As of 2014, an estimated 1,800 Mendelian conditions remain to be identified (http://www.omim.org/statistics/ entry). We recently have established a research program (www.tidebc.org) using whole exome sequencing (WES) in patients with unexplained metabolic phenotypes to diagnose/discover inborn errors of metabolism as a potential

\footnotetext{
* Correspondence: sstockler@cw.bc.ca

${ }^{\dagger}$ Equal contributors

'Division of Biochemical Diseases, B.C. Children's Hospital, Vancouver, Canada 2Department of Pediatrics, University of British Columbia, Vancouver, Canada Full list of author information is available at the end of the article
}

cause of their intellectual disability and/or developmental delay [1]. Here we report such a case, in which a mutation in the gene ZFYVE20, encoding the protein Rabenosyn-5 (Rbsn-5), is the likely cause of a complex condition in a female child characterized by developmental delay, intractable epileptic encephalopathy, and a pleiotropic clinical and biochemical phenotype.

Rbsn-5 is a large, evolutionarily conserved multidomain protein expressed ubiquitously in mammalian cells and implicated in receptor-mediated endocytosis [2-5]. The specific role of Rbsn- 5 is to regulate the intracellular route of internalized receptors, facilitating their recycling to the 
plasma membrane [5-7]. By controlling the rate of recycling of numerous receptors, Rbsn-5 affects critical cellular functions, amongst which are the absorption and cellular uptake of high molecular weight nutrients, the signaling capacity of growth factor and neurotransmitter receptors, and the ability of integrins to control cellular motility. At the molecular level, Rbsn-5 controls receptor recycling $[5,7]$ through direct interactions with regulatory proteins and lipids, which include the endocytic GTPases Rab4 and Rab5, and phosphatidylinositol-3 phosphate, the product of the class III PI-3 kinase Vps34 [6,8-10]. Thus, mutations that cause structural alterations in specific regions of Rbsn-5 are likely to disrupt the balance of interactions with one or more of these regulatory factors, and lead to widespread abnormalities in receptor recycling dynamics.

Transferrin (Tf) is frequently used to monitor endocytosis and recycling, due to the abundance of transferrin receptors in primary and cultured cells, as well as the property of $\mathrm{Tf}$ to become internalized and quantitatively recycled. Rbsn-5 depletion in cultured mammalian cells impairs Tf trafficking [5-7] by interfering with recycling, and can lead to decreased receptor levels [5]. High-resolution live cell imaging studies have shown that Rbsn-5 is localized to endosomes to which Tf is delivered immediately following its internalization from clathrin-coated pits at the plasma membrane [5].

We have used this approach to determine how the mutation in Rbsn-5 in this patient impairs endocytosis and recycling. Additional biochemical and morphological approaches have been used to further understand the pleiotropic cellular consequences of this disruption. Possible mechanisms by which these defects culminate in the overall clinical presentation in this patient are discussed.

\section{Methods}

This study was initiated as part of the Treatable Intellectual Disability Endeavor in British Columbia and approved by the institutional review boards of BC Children's Hospital and the University of British Columbia (CW12-0019/ H12-00067). Parents provided written informed consent.

\section{Whole exome sequencing}

Index and unaffected parental sequencing was conducted using Agilent SureSelect kit and Illumina HiSeq 2000 (Perkin-Elmer, Santa Clara, California, USA) (REB Approval CW12-0019 / H12-00067) Approximately 50.2 million 100 bp pair-end reads were generated per participant. A combination of Bowtie, BWA and GSNAP was used to map the reads to hg19 reference genome, and Samtools was used to call the variations relative to the reference. On average, 99\% of the observed variations were classified as common variants based on a reported allele frequency in dbSNP (version 135). The remaining set of rare or novel variants (defined as $<0.01$ minor allele frequency) was assessed for potential to disrupt protein function using the Sift and PolyPhen2 software systems, and was also screened under a series of genetic models with a focus on Mendelian recessive modes of inheritance.

\section{PCR}

Exome sequencing results of the mutations in ZFYVE20 were validated in the index and unaffected parents (with targeted mutation analysis in the unaffected siblings), Sanger DNA sequencing using standard PCR conditions with an annealing temp of $59^{\circ} \mathrm{C}$. (primer 1: GGGTCTG AGTCCTCACTCTGC, primer 2: TGTCACTGGCACAG GGATAG).

\section{Cell culture and transfection}

$\mathrm{CB}$ and PA cells were maintained in MEM 2\% FBS. Cells were transfected using electroporation, plated on glass coverslips (Thomas Scientific 25 circle \#1.5), and grown for 48 hours. Live imaging was done in $\mathrm{KRH}$ buffer (125 M NaCl, $5 \mathrm{mM} \mathrm{KCl,} 1.3 \mathrm{mM} \mathrm{CaCl}_{2}, 1.2 \mathrm{mM} \mathrm{MgSO}_{4}$, $25 \mathrm{mM}$ HEPES, $2.5 \% \mathrm{BSA}$ and $2 \mathrm{mM}$ sodium pyruvate) $\mathrm{pH} 7.4$.

\section{TIRF/Epi-fluorescence structure-illumination microscope (TESM) optical system}

A custom-built microscope system, TESM, simultaneously combines Total Internal Reflection Fluorescence and wide-field epifluorescence modes and incorporates structured illumination in the epi mode for fast optical sectioning and enhanced spatial resolution. Further details as well as the TESM acquisition system is described previously [5]. For quantification, image sets were convolved with a difference of Gaussians (DOG) filter consisting of 1) a small, two dimensional, Gaussian spot with unit area $($ sigma $=150 \mathrm{~nm})$ that acted as a vesicle matched detector, i.e. an approximation to a near-diffraction limited spot, and 2) a larger, inverted, two dimensional Gaussian $($ sigma $=300 \mathrm{~nm})$ with negative unit area that estimated and subtracted the local background. The Gaussian smoothed images were visually thresholded (global threshold) to select for pixels belonging to objects (e.g. vesicles) and eliminate areas devoid of signal (but containing noise). The total intensity per cell over time was recorded.

\section{Reagents}

The TagRFP-T expression vector was constructed as described [11]. The cDNA clone 40034008 for human Rabenosyn-5 was obtained from American Tissue Culture Collection (Manassas, VA) and was cloned in frame with TagRFP-T at the $\mathrm{N}$ terminus of the protein using standard techniques. Polyclonal EEA1 and Rbsn-5 antibodies have been described [5]. Unconjugated and DyLight-conjugated human transferrin were obtained from Jackson Immunochemicals. 


\section{Electron microscopy}

Fibroblasts were fixed and postfixed in phosphate buffered $2.5 \%$ glutaraldehyde and osmium tetroxide respectively, dehydrated in an ascending series of acetone and infiltrated and embedded in epon araldite, and ultrathin sections were prepared and mounted on grids prior to examination in the electron microscope.

\section{Cathepsin D analysis}

Goat anti-human CatD antibody was used to visualize the $53-\mathrm{kDa}$ proenzyme precursor (preCatD) and the processed $33 \mathrm{kDa}$ heavy chain (CatD) of cathepsin D by western blotting. Blots were stained with Lumi Light Western blotting substrate (Roche Diagnostics, Mannheim, Germany) to visualize the $53-\mathrm{kDa}$ proenzyme precursor (preCatD) and the processed $33 \mathrm{kDa}$ heavy chain (CatD) of cathepsin $\mathrm{D}$ by chemiluminescence [12].

\section{Results}

\section{History and physical exam}

This 6.5 year-old girl was born as the second of 3 sisters to healthy non-consanguineous Caucasian parents after an uneventful pregnancy. She developed pharmacoresistant infantile spasms at age 5 months, which improved upon ketogenic diet (KD) started at age 14 months. Severe hypertriglyceridemia (plasma triglycerides: $90 \mathrm{mmol} / \mathrm{L}$, normal $0.36-1.31$ ) observed at age 50 months was reversible by reduction of natural fat in the KD, and partial replacement with medium chain triglycerides. Her cranial 1.5 T MRI, at age 40 months showed moderate enlargement of the $3^{\text {rd }}$ ventricle, normal myelination and cortical architecture.

At $6.5 \mathrm{y}$ she is clinically seizure free upon the modified KD and four anticonvulsive drugs (Valproic acid, Phenobarbital, Levetiracetam, Lamotrigine). Her biparietal head circumference is $46 \mathrm{~cm}$ (below the $0.1^{\text {st }}$ percentile); weight and height at the $3^{\text {rd }}$ and $0.1^{\text {st }}$ percentile. She is hypotonic, able to sit without support, but unable to stand or walk. She has a happy, friendly demeanor, is non-verbal and unable to feed herself and not toilet-trained. She has thin, whispy hair and dysmorphic facial features (Figure 1A).

A skeletal survey at chronological age 6 years revealed a moderate osteopenia involving the pelvis and long bones of both upper and lower limbs with evidence of undertubulation and hypoplasia of the epiphyses around the knee joint and bilateral coxa valga. Particularly the long bones of the right hand appear undertubulated and osteopenic. (Figure 1B) with delayed appearance of the right carpal bones (approximate bone age 2.5 years).

\section{Clinical biochemistry}

At 14 months of age (prior to start of the ketogenic diet), biochemical genetic assessment revealed repeatedly normal results for blood lactate and ammonia levels,
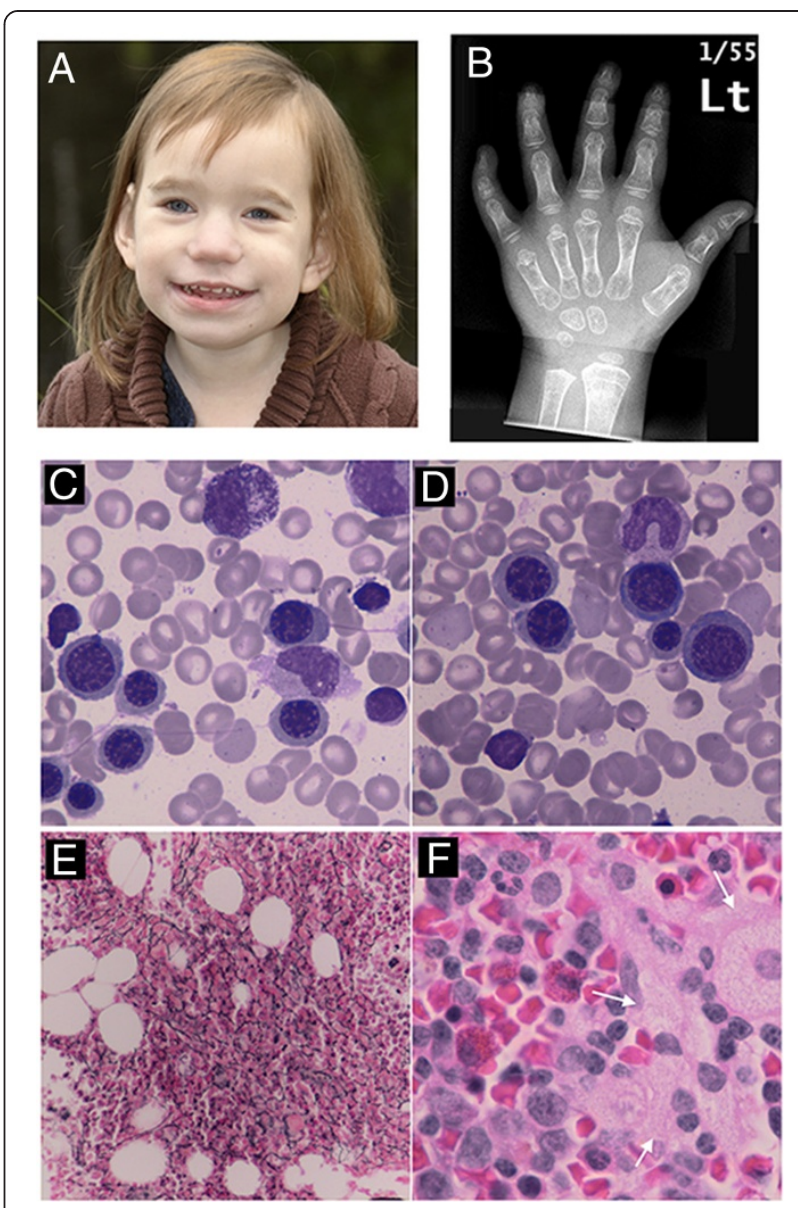

Figure 1 Clinical features of a girl with a point mutation in Rabenosyn-5. A: Facial features showing midfacial bone hypoplasia, deep set eyes with a hooded appearance, a fullness in the nasal bridge, a short nose and a large mouth with small teeth and tongue protrusion. B: PA view of right hand showing marked under mineralization, coned epiphyses, under tubulated long bones, and relatively short digits particularly involving the middle phalanges of digit 2 and digit 5. Delayed skeletal maturation at approximately 2 years 6 months for a chronological age of 6 years 2 months and a single standard deviation of 10 months. C-F: Bone marrow aspirates showing megaloblastoid erythropoiesis (C and $\mathbf{D}$ ), occasional intercytoplasmic bridging $(\mathbf{C})$, mild increase in reticulin fibrosis throughout most of the bone marrow (E). Foamy macrophages most notably in the bone marrow biopsy specimen (F).

plasma and CSF aminoacids, plasma very long chain fatty acids, pipecolic acid, and transferrin phenotyping, urine excretion of purines and pyrimidines, creatine, guanidinoacetate, glycosaminoglycans, oligosaccharides and $\alpha$-aminoadipic acid semialdehyde. Urinary organic acid analysis revealed high urine methylmalonic acid concentration ( $40 \mathrm{mmol} / \mathrm{mol}$ creatinine, normal $<5.13)$ along with mildly elevated plasma homocysteine $(13.7 \mu \mathrm{mol} / \mathrm{L}$, normal $<7$ ) and low cobalamin (vitamin B12) levels (66 and $60 \mathrm{pmol} / \mathrm{L}$, deficient range $<107)$. The child was breastfed until age 14 months when she started the ketogenic diet. The mother had been on a normal, vitamin 
B12 containing diet throughout pregnancy and the time of breastfeeding. At the time vitamin B12 deficiency was diagnosed in her child, her plasma vitamin B12 levels were within non-deficient range $(178 \mathrm{pmol} / \mathrm{L}$, normal 156-698), her plasma homocysteine level was $13 \mu \mathrm{mol} / \mathrm{L}$ (normal 6-12.8), her CBC, urine organic acid profile and blood acylcarnitine profile were normal. Cobalamin levels in the child increased significantly (up to $1107 \mathrm{pmol} / \mathrm{L}$ ) after intramuscular injection of vitamin B12 (cyanocobala$\min 1000 \mu \mathrm{g}$ ) on 10 subsequent days, and remained within non-deficient range $(471 \mathrm{pmol} / \mathrm{L}$, non deficient range: 133-675) upon continuous oral supplementation of cyanocobalamin $(250 \mu \mathrm{g}$ per day) when measured at 26 months of age. Homocysteine remained normal when measured at various occasions $(3.1,5.0,2.5 \mu \mathrm{mol} / \mathrm{L})$, but urine and serum MMA levels remained mildly elevated when measured at 29 and 40 months of age (urine: 31 and $32 \mathrm{mmol} /$ mol creatinine, normal $<5.13$; serum: 0.21 at 40 months, normal 0.018-0.150).

Defects of cobalamin absorption, transport and intracellular metabolism were excluded by respective tests in blood and cultured fibroblasts (Table 1). Also detected were microalbuminuria (microalbumin/creatinine ratio = 15.6 - 26.8, normal <2.7) and high urinary Ca excretion (calcium/creatine ratio $=3.73$, normal $<1.1$ ) in the presence of normal plasma $25 \mathrm{OH}$ Vitamin D, calcium, phosphorus and alkaline phosphatase levels.

\section{Clinical hematologic findings}

Red blood cell macrocytosis (range: 95.5-104.6 fl; mean: 98.8; $\mathrm{n}=21$; normal $75-87$ ) was noted prior to initiation of the ketogenic diet and persisted throughout the entire observation period. Transient neutropenia occurred between age 40 and 44 months (range: $0.7-1.3 \times 10^{9} / \mathrm{L}$, mean: $0.9, \mathrm{n}=4$; normal: $1.5-8.5)$. A bone marrow aspirate and biopsy at age 44 months showed normocellularity for age with a normal myeloid to erythroid ratio (2:1) (Figure 1 C-F). A mild increase in reticulin fibrosis (2+ out of 4) but no collagen deposition were present. No significant dysplastic features were seen in the granulocytic and megakaryocytic lineages. Foamy macrophages were attributed to the marked hyperlipidemia present at the time of bone marrow sampling. Peripheral blood morphology at the time of bone marrow biopsy showed increased target cells and stomatocytes. Erythropoiesis showed megaloblastoid change with some erythroblasts showing features approaching a true megalosblastic state, however no neutrophil hypersegmentation nor giant myelocytes or metamyelocytes were present and iron stains did not show ring sideroblasts. Cytogenetic studies were unremarkable at the time. Plasma copper, zinc and folate, erythrocyte folate, serum iron and ferritin and transferrin saturation were normal on repeated occasions.

\section{Genetic analyses}

The patients karyotype and chromosomal microarray analysis (AffymetrixCytoscan ${ }^{\bullet}$ HD) were unremarkable; homozygosity analysis did not reveal evidence of consanguinity or uniparental disomy. WES was performed for the index and her unaffected parents. Rare variants were assessed for their potential to disrupt protein function and screened under a series of genetic models-primarily the

Table 1 Studies of cobalamin absorption, transport, cellular uptake and intracellular metabolism in a patient with a homozygous G425R mutation in ZFYVE20

\begin{tabular}{|c|c|}
\hline Intrinsic Factor antibodies & Negative antibodies \\
\hline \multicolumn{2}{|l|}{ Parietal Cell antibodies } \\
\hline Gastric Intrinsic Factor Protein (GIF) & No pathogenic mutations on WES (coverage $\times 30$, last 5 exons of AMN $\times 3-5$ ) \\
\hline \multicolumn{2}{|l|}{ Cubilin (CUB) } \\
\hline \multicolumn{2}{|l|}{ Megalin (LRP2) } \\
\hline \multicolumn{2}{|l|}{ Amnioless (AMN) } \\
\hline Total Haptocorrin (TCN1) & 592 pmol/L (normal 240-680) \\
\hline Plasma Total Transcobalamin (TCNII)* & 1520 pmol/L (normal 500-1.500) \\
\hline Plasma Holotranscobalamin* & 182 pmol/L (normal 40-160) \\
\hline \multirow[t]{2}{*}{ Transcobalamin Receptor related genes (TCbR, CD320, TCN1, TCN2) } & Absence of (262_264delGAG and 297delA) by targeted mutation analysis [17] \\
\hline & No pathogenic mutations by WES (coverage $>\times 20$ ) \\
\hline$\left[{ }^{14} \mathrm{C}\right]$-methylene tetrahydrofolate incorporation (f) & 250 pmol/mg protein/18 h (normal $225 \pm 165$ pmol/mg protein/18 h) \\
\hline$\left[{ }^{57} \mathrm{Co}\right]$-cyanocobalamin uptake $(\mathrm{f})$ & $16.9 \mathrm{pg} / 10^{6}$ cells (normal $13.2 \pm 4.8 \mathrm{pg} / 10^{6}$ ) \\
\hline$\left[{ }^{57} \mathrm{Co}\right]$-cyanocobalamin conversion to adenosylcobalamin ( $f$ ) & 5.7. \% of intracellular cobalamin (normal $15.3 \pm 4.2$ ) \\
\hline$\left[{ }^{57} \mathrm{Co}\right.$-cyanocobalamin coversion to methylcobalamin (f) & $53.0 \%$ (normal $58.0 \pm 6.7$ ) \\
\hline
\end{tabular}

(f) = fibroblasts; *by courtesy Ebba Nexo, Arhus, DK. 
Mendelian recessive mode of inheritance given the rarity of the phenotype and the pattern of inheritance of most IEMs.

Approximately $99 \%$ of the observed variations were classified as common (results not shown). Two rare (defined as $<0.01$ minor allele frequency) candidate variants fit the compound heterozygous model autosomal recessive model of homozygous WES revealed a total of 5 genes harboring compound heterozygous variants and 3 genes with homozygous variants (SLC41A3, CLIP, ZFYVE20) Only the homozygous missense variation (g. 15,116,371 $\mathrm{G}>\mathrm{A}$ ) in ZFYVE20, resulting in a glycine to arginine substitution at position 425 (p.Gly425Arg), segregated with disease in the family as confirmed by Sanger sequencing (Figure 2).

\section{Cellular endocytosis and recycling analysis}

The uptake and recycling rates of transferrin (Tf) in patient (PA) and age and passage matched control (CB) fibroblasts were studied using described methods $[5,13]$. In brief, cells were imaged by total internal reflection fluoresce microscopy (TIRF) at 1 frame/sec continuously for 20 minutes, during which Alexa-488-Tf was added to the medium, maintained for 10 minutes and then removed. Raw TIRF images obtained over time from control (Figure 3A) or patient (Figure 3B) single cells, together with mean and SEM from three independent cells are illustrated in Figure 3C. Data normalized to the maximal uptake recorded in each cell is shown (Figure 3D).

Tf associated rapidly and saturably with both PA and $\mathrm{CB}$ cells, displaying kinetic constants consistent with binding to the transferrin receptor [13-16], and similar to those previously determined in other mammalian cell types. However, in PA cells Tf saturated at a lower maximal rate (Figure $3 \mathrm{C}$ ), and displayed a significantly faster rate of recycling compared to $\mathrm{CB}$ cells (Figure 3C,D,G). The accumulation of $\mathrm{Tf}$ in the three dimensional volume of the cell was visualized immediately before the removal of extracellular Tf, by collecting 10 image stacks using structured light illumination. Accumulation of Tf in PA cells was only $50 \%$ of that observed in CB cells (Figure 3E,F). Results demonstrate a defect in the early endocytic pathway with enhanced recycling rate and decreased steady-state accumulation of ligand in cells harboring the p.G425R mutation.

Western blotting did not show significant differences between PA and CB total cellular levels of Rbsn-5 and other key proteins within the early endocytic pathway (Figure 4A), ruling out the possibility that the p.G425R mutation might result in loss of protein stability and enhanced degradation. Moreover, no consistent difference in the total level of the transferrin receptor (TfR) was noted, suggesting that the alterations in Tf endocytosis in PA cells are not simply a consequence of lower

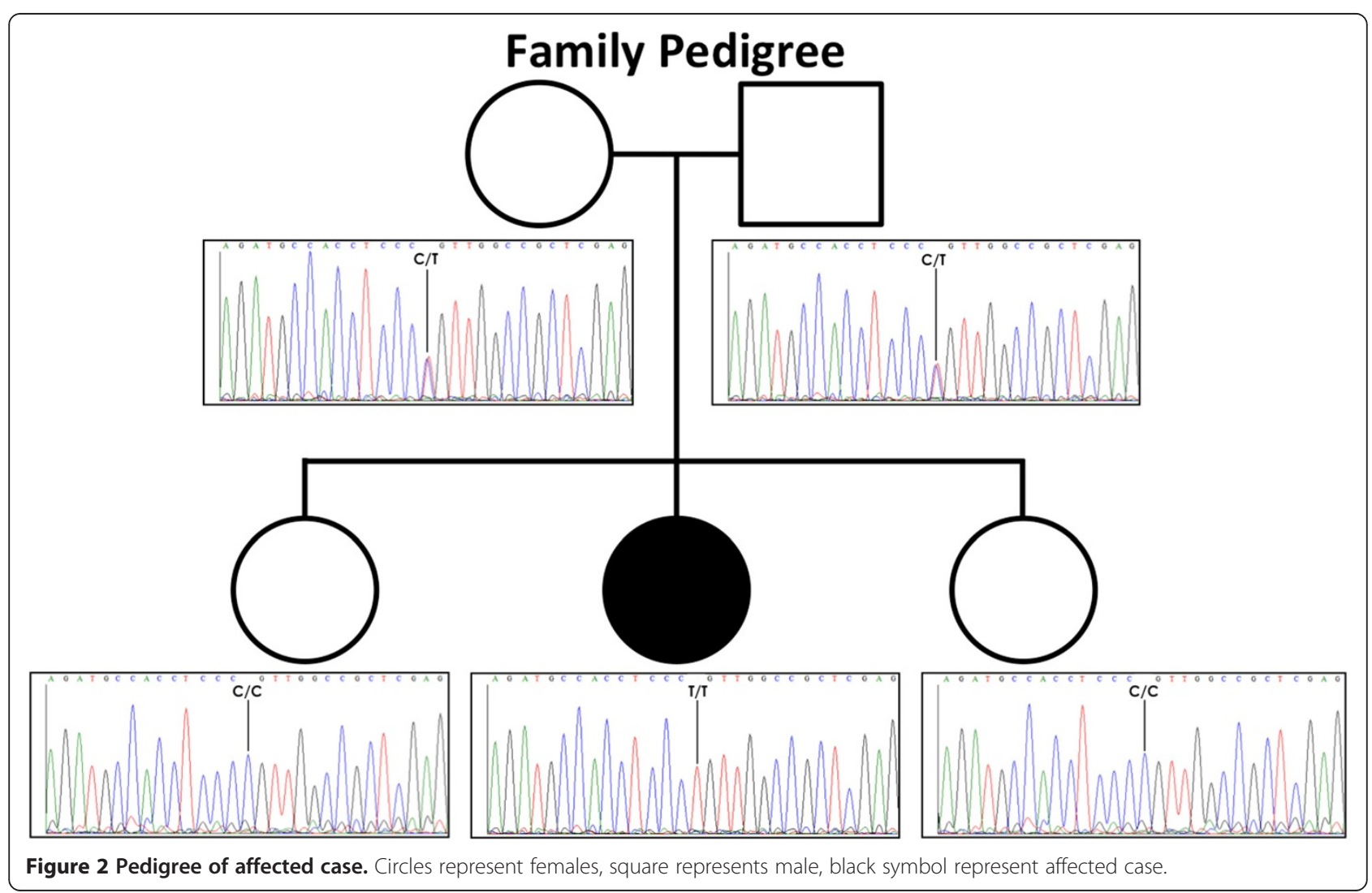



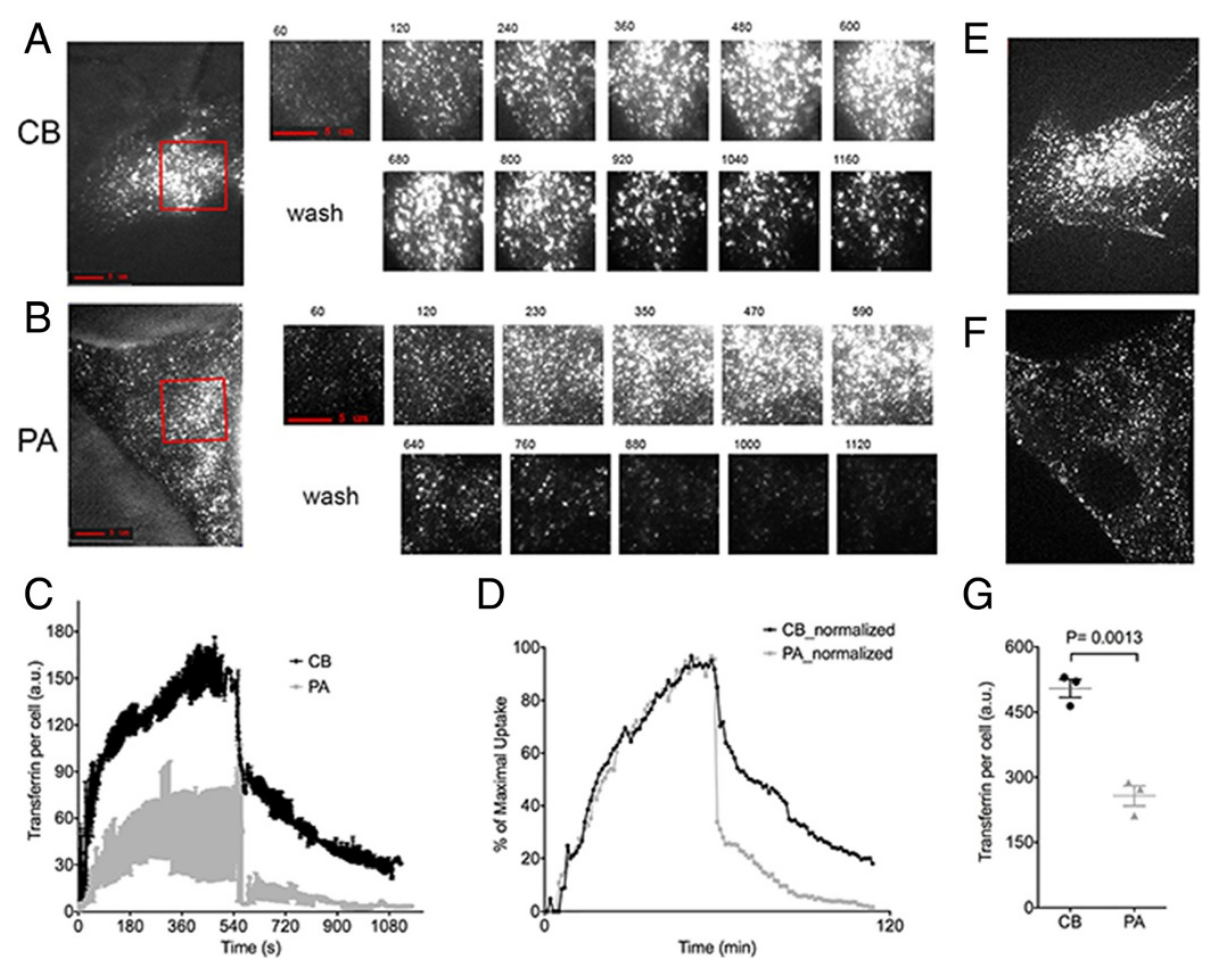

D

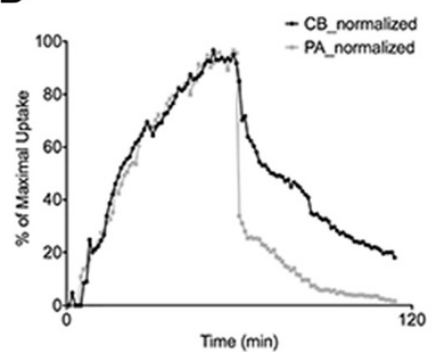

$\mathrm{G}$

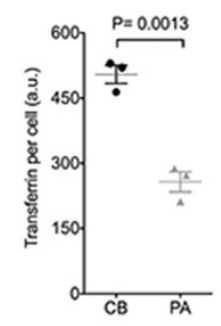

Figure 3 Dynamics of transferrin association and dissociation from human fibroblasts. TIRF images of the entire cell from control (A) or affected (B) individual at 300 seconds following addition of fluorescent transferrin. Red rectangles represent region depicted in the time series shown to the right. Transferrin was removed from the medium at $t=600$. C. Quantification of the amount of transferrin in the TIRF image of the entire cell, plotted as the mean and SEM of each time point from three individual cells. D. The mean from the data in C was recalculated as a percent of maximal signal. Structured light epifluorescence image stacks from control $(\mathbf{E})$ or affected $(\mathbf{F})$ individual taken at $t=600$, projected into a single plane. G. Quantification of signal in the structured light images from 3 individual cells. Statistical significance was calculated by 2-tailed Student t-test. Similar experiments were conducted with three independent passages, with similar results.

receptor levels. Immunofluorescence staining with antibodies to Rbsn-5, EEA1, clathrin and TfR showed similar distribution in CB and PA cells and comparable to that previously described for Cos-1 cells (Figure 4B).

Finally, we investigated restoration of function transfecting PA and BC cells by electroporation with either a control plasmid encoding soluble RFP or RFP-Rbsn-5.
After $48 \mathrm{~h}$, cells were exposed to Alexa488-Tf for the indicated time (Figure 5) and accumulation per cell was measured. CB fibroblasts expressing RFP (Figure 5A,C) or RFP-Rbsn-5 (Figure 5B,D) accumulated a similar amount of Tf over different time points analyzed (Figure 5C,D and I). In contrast, Tf uptake into PA cells was lower than that seen in CB cells, and significantly increased upon expression

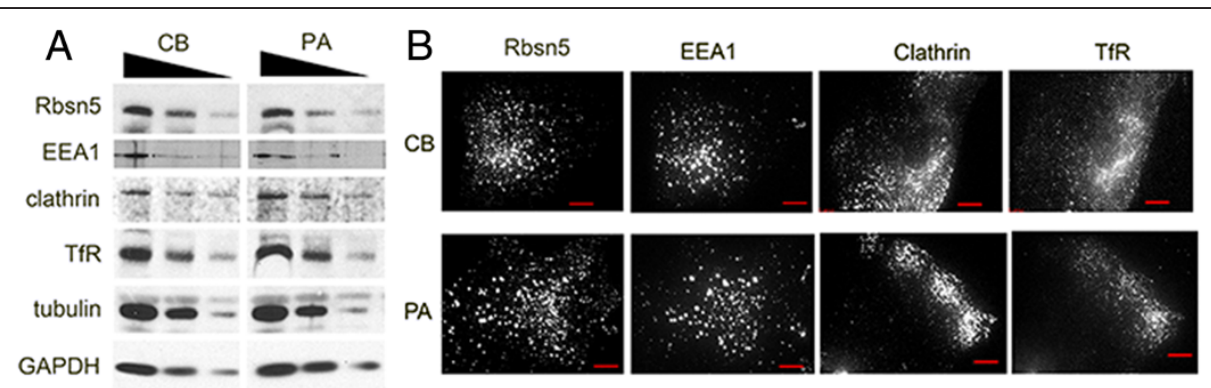

Figure 4 Western blotting and subcellular distribution of endocytic pathway proteins. A. Western blotting: Cells from control (CB) or affected (PA) individual were grown to $80 \%$ confluence, and whole cell lysates obtained. Serial dilutions of equal total protein concentrations of the cell extracts were processed to enable quantification, as indicated. B. Subcellular distribution: Cells from control (CB) or affected (PA) individual were grown on glass coverslips, fixed, permeabilized and stained with antibodies to the proteins indicated above each panel. Shown in black and white are structured light epifluorescence image stacks from representative cells, projected into a single plane. 


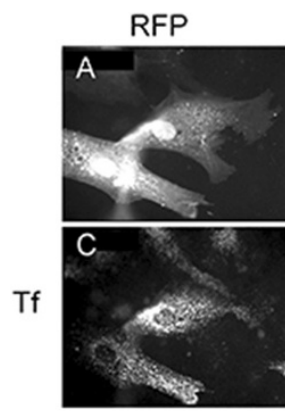

RFP
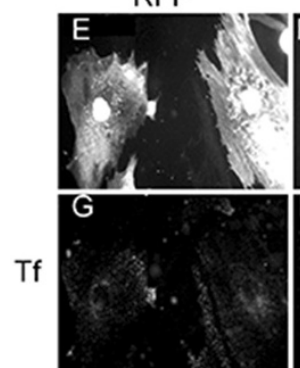

RFP-Rbsn5

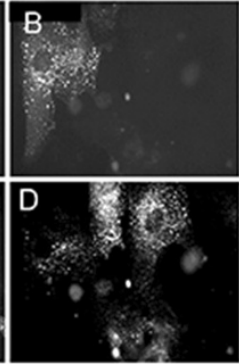

RFP-Rbsn5

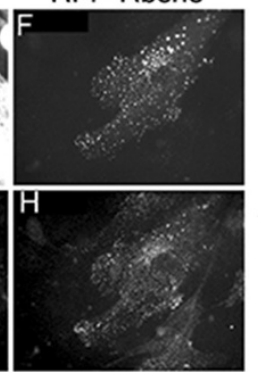

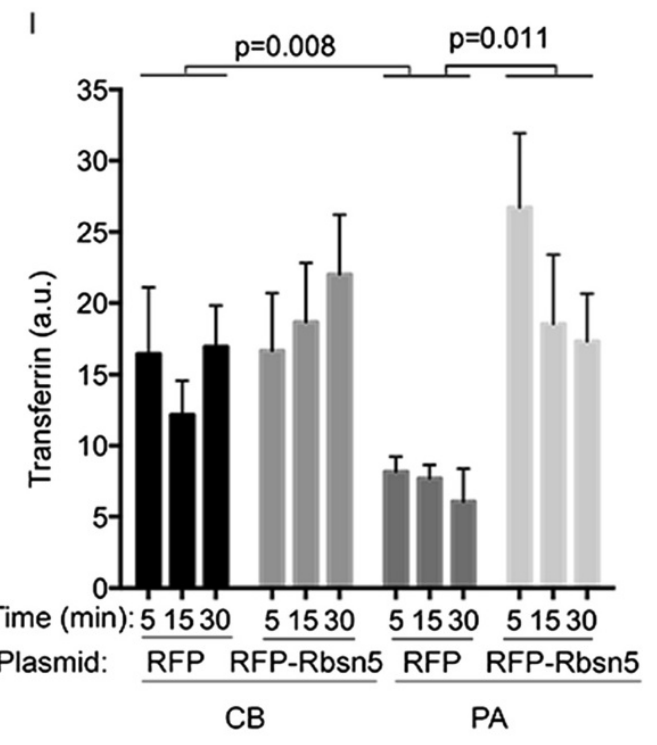

PA

Figure 5 Rescue of Tf accumulation by wild-type Rbsn-5. Cells from CB (A-D) or PA (E-H) were transfected with plasmids encoding RFP (A,C,E,G) or RFP-Rbsn5 (B,D,F,H). After 48 h, cells were exposed to Alexa-488-Transferrin for 5-30 min, washed briefly and fixed. Representative images of RFP (A,E) or RFP-Rbsn5 (B,F) and corresponding Tf images (C,G and D,H). Light intensity of Tf signal per cell was quantified (I). Plotted are means and SEM of 50-100 cells from 4 independent experiments. Statistical significance was calculated using 2-tailed paired Student t-test, where the means of each time point are paired as indicated.

of RFP-Rbsn-5 (Figure 5G,H and I). Experiments suggest that the observed defect in transferrin trafficking is attributable to the Rbsn-5 G425R mutation.

We then tested whether the effect of the Rbsn-5 p. G425R mutation might be restricted to Tf recycling kinetics, or as expected, would affect the activities of other receptors. Depletion of Rbsn-5 causes altered subcellular distribution of mannose-6-phosphate receptors affecting the biosynthetic transport of cathepsin D from Golgi to the lysosomal lumen [17]. Consistent with this function, we found a reduction of cathepsin D activity to $35 \%$ of the one measured in control fibroblasts $(404 \pm 53$ versus $1158 \pm 178 \mathrm{nmol} / \mathrm{h} \times \mathrm{mg}, \mathrm{n}=3$ ). In Western blotting, the intensity of precursor cathepsin $\mathrm{D}$ was slightly higher in the patient fibroblast, in contrast the intensity of $33 \mathrm{kDa}$ heavy chain of the mature cathepsin $\mathrm{D}$ was diminished to $42 \%$ of the control (Figure 6A), indicating compromised cathepsin $\mathrm{D}$ processing to mature, proteolytic active cathepsin D. Targeted WES analysis of CTSD (with $\geq 25 \mathrm{X}$ coverage of all exons) did not identify any rare, damaging variants.

To further assess the consequences of impaired trafficking, growth rates and subcellular morphology of the PA cells were analyzed. A slower proliferation rate was found (Figure 6B), as well as the presence of translucent vacuoles in the perinuclear region (Figure $6 \mathrm{C}$ ). These vacuoles were negative for Tf even after prolonged uptake, and negative for lysotracker staining (not shown). The presence of vacuoles was not mitigated by transient expression of Rbsn-5, and thus may be the result of persistent changes caused by endocytic pathway abnormalities, which are not reversible in a short-term experiment. Electron micrographs of the patient's fibroblasts (Figure 6D-F) showed an excess of intermediate filaments in either a perinuclear band, associated with the plasmalemma, or throughout the cytoplasm, as well as lysosomes containing small granules of electron opaque material. Cytoplasmic organelles were sparse when compared to fibroblasts derived from normal individuals.

\section{Discussion}

Here we report a complex clinical phenotype associated with a mutation in exon 14 of ZFYVE20, leading to mutation of glycine 425 to arginine in Rbsn-5, which appears to affect the dynamics of receptor trafficking in the endocytic pathway. To our knowledge this is the first report of mutations in this highly conserved multidomain protein, and the complex ensuing clinical phenotype is consistent with the fundamental role of endocytosis and recycling in mammalian cell function, and with the critical role that Rbsn-5 plays in this multistep process.

The location of this mutation is in the central residue in an NG(D/E) motif that stands out as an island of conservation within an otherwise variable segment in higher metazoans (mammals, birds, fish, reptiles) (Figure 7). We are currently investigating the effect of this mutation 


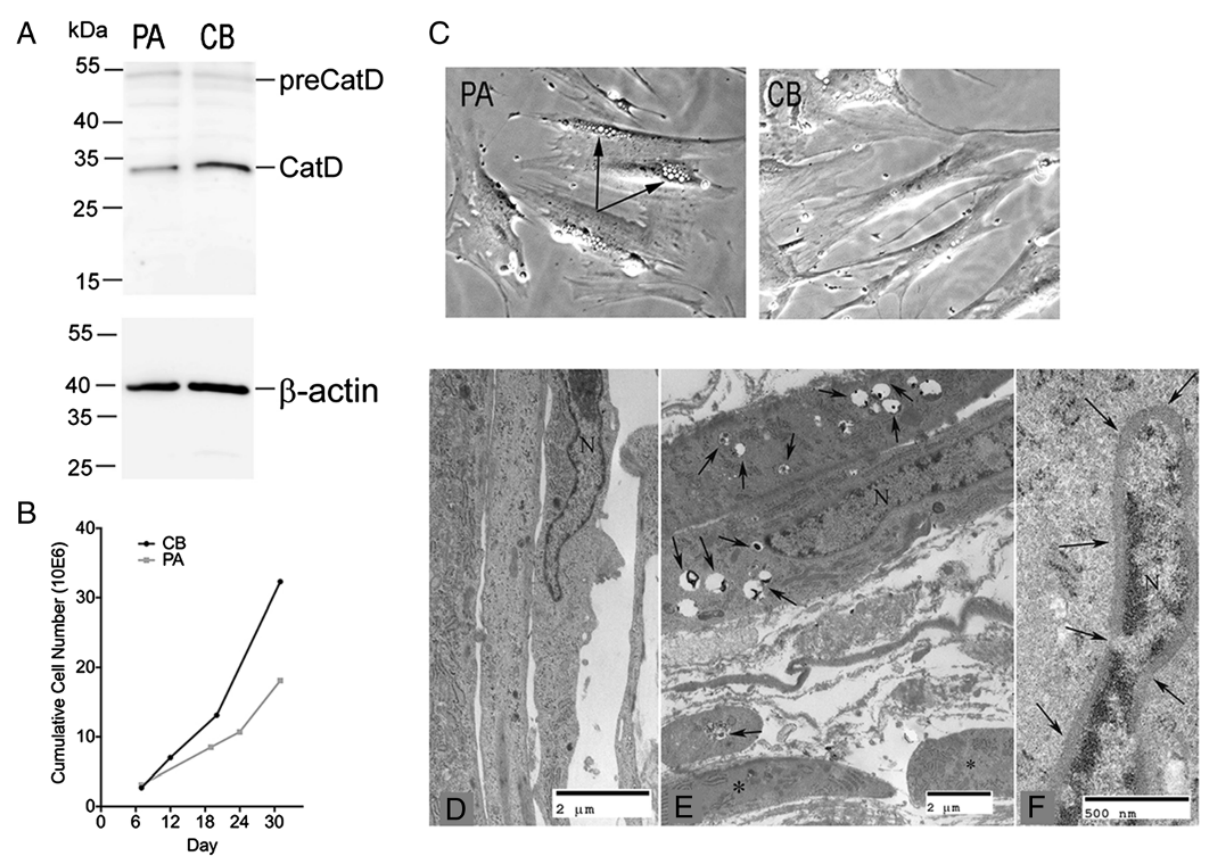

Figure 6 Additional findings in fibroblasts A. Western blot of extracts from cells from control (CB) or affected (PA) individual with goat anti-human CatD antibody, indicating 53-kDa proenzyme precursor (preCatD) and the processed $33 \mathrm{kDa}$ heavy chain (CatD) of cathepsin-D. Detection of $\beta$-actin demonstrated similar amounts of protein loading for patient and control lysates. B. Cumulative cell numbers calculated at each passage over a 30 day period. C. Phase images of CB (left) and PA (right) fibrobasts. Arrows indicate translucent vacuoles present in many of the PA cells. Electron micrographs of normal (D) and patient (E) fibroblasts. F. Nucleus (N) from patient's fibroblast surrounded by a dense band of filamentous material.

on the tertiary structure of the protein using X-ray crystallography and small angle X-ray scattering. Mutation to arginine as found in this patient, represents a major, non-conservative change that could result in loss of function with respect to flexibility, structural organization, and/or intermolecular interactions, in addition to potential effects on membrane targeting, stability, solubility, or oligomeric state. These alterations are likely to affect the function of Rbsn-5 to generally control receptor trafficking, which in turn would affect the levels and activity of multiple receptor species in multiple cell types, thus underlying multi-organ level abnormalities. Consistent with this possibility, we find evidence for enhanced receptor recycling when directly visualizing Tf endocytosis, as well as reduced residual cathepsin D activity with predominance of pre-cathepsin $\mathrm{D}$ protein, indicating

\section{A

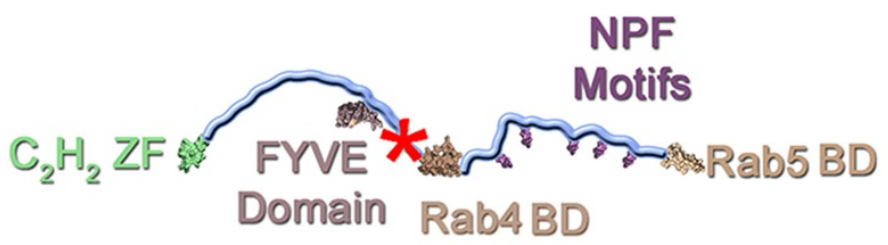 \\ B

Pred. Helix
hs

Figure 7 Position of G425R mutation in the context of full-length Rbsn-5. A. Diagram of Rabenosyn-5 with functional domains indicated. Red asterisk indicates position of mutation. B. Amino acid conservation among species in the region of the mutation. 
impaired trafficking of this enzyme in the endolysosomal system. These phenotypes have been previously associated with decreased levels of Rbsn-5 achieved through siRNA silencing [5,17]. Understanding how the G425R mutation phenocopies Rbsn-5 depletion will require further structure-function studies outside the scope of the present findings.

The mechanisms by which deficiency of Rbsn- 5 at the cellular level lead to the complex features of this patient are suggested by cases in which specific defects in the endosomal/lysosomal pathway produce complex clinical phenotypes. For example, primary cathepsin D deficiency $[12,18]$, causes early onset epileptic encephalopathy and microcephaly, features, which are prominent in our patient's clinical phenotype. Similarly, mucolipidosis type III, a lysosomal trafficking disorder caused by loss of mannose-6-phosphate targeting signals on lysosomal proteins, results in dysmorphic facial features, small stature and dysostotic bone changes similar to those observed in our patient $[19,20]$. The electron-dense material contained in the lysosomes of this patient's fibroblasts may indicate lysosomal storage due to reduced intralysosomal amounts of enzymes dependent on mannose-6phosphate mediated trafficking $[19,20]$.

Alterations in endocytic trafficking caused by Rbsn- 5 deficiency might be the common denominator for the pleiotropic clinical manifestations observed in this patient. For example, the severe dyslipidemia developed in response to ketogenic diet may in part be attributable to impaired LDL receptor trafficking. While the child's initial vitamin B12 deficiency might in part be of nutritional origin, in combination with the observed albuminuria, impaired endocytic trafficking in proximal renal tubular cells, where transcobalamin and albumin are reabsorbed by the Megalin/cubilin receptor protein [21], might have been an aggravating factor. Likewise, the urine methylmalonic acid excretion and the red blood cell macrocytosis, despite cobalamin supplementation, may be due to defective intracellular trafficking of cobalamin. Although not entirely explained, the disproportionate synthesis of methylcobalmin and adenosylcobalamin in the patient's fibroblasts might be an indication of this proposed mechanism as well.

There is also a possible relation between the observed bone marrow changes and Rbsn- 5 deficiency. Missense mutations leading to depletion of Vps45, a known Rbsn5 binding partner, have recently been reported in patients with severe neutropenia, bone marrow fibrosis and early lethality $[22,23]$. The reticular fibrosis found in our patient's bone marrow resembles these findings. Functional folate deficiency caused by folate antagonistic anti-epileptic drugs (e.g. lamotrigine) and the KD also could explain these findings, although hematological studies proving such side effects are lacking.
The cumulative effects of Rbsn- 5 deficiency on cellular function are manifested by a slow growth rate of the patients fibroblasts, compared to age matched controls, as well as by morphological abnormalities such as the accumulation of translucent vacuoles and intermediate filaments. The extent to which these abnormalities are directly attributable to Rbsn-5 function, or are secondary to chronic impairment of endocytic trafficking is unclear, but the failure of Rbsn-5 wild-type transfection to revert vacuolar phenotype is suggestive of the latter possibility. Nevertheless, impaired cytoskeletal dynamics have been seen in models of Rbsn-5 deficiency [24], and could explain the accumulation of intermediate filaments in the perinuclear cytoplasm of our patient. While changes in neurotransmitter recycling [25-27], cytoskeletal dynamics [28], or dendritic branching [29] are possible causes, the precise mechanism by which this mutation contributes to the pathogenesis of epilepsy, a key feature in this patient, remains to be determined.

\section{Conclusions}

Overall, based on the critical role of Rbsn-5 in endocytic pathways, mutations affecting its functions are likely the basis of multi systemic disorders such those presented here. More patients with Rbsn-5 deficiency need to be identified in order to delineate the specific clinical and biochemical phenotype of this gene defect. Patients with epilepsy, growth retardation, dysmorphic features, multisystem involvement (bone abnormalities, myelodyplasia, albuminuria) and unexplained biochemical findings such as cobalamin deficiency and partial cathepsin D deficiency should be considered at risk.

\section{Competing interests}

The authors declare that they have no competing interests.

\section{Authors' contributions}

SS: selected the patient for exome sequencing, directed and interpreted phenotypic analyses, drafted the manuscript. SC: directed phenotypic analysis of cell growth, Tf trafficking, rescue by wild-type allele, and immunofluorescence localization of proteins, drafted the manuscript. DL: analyzed consequences of mutation on protein structure. KF: Developed software and hardware microscopy platform for phenotypic characterization of cells. DL: conducted phenotypic analysis of cell growth, Tf trafficking, rescue by wild-type allele, and immunofluorescence localization of proteins. CA: performed electrone microscopic studies. RS: performed Cathepsin D studies. EN, CS, WW: performed the bio-informatics interpretation of WES data. NA: performed hematologic studies in bone marrow biopsy. RW: contributed to biochemical phenotyping. KS: directed clinical care of the patient. MA: performed medical genetic studies. HV: directed biochemical genetic studies. DR and DW:

performed and interpreted cobalamin studies. CR: performed and interpreted the Sanger sequencing studies. EC: contributed to the design and the interpretation of the in vitro studies, edited the manuscript. CK: coordinated the phenotypic analyses, collected the clinical data and coordinated the genetic/genomics analyses, coordinated the drafting and editing of the manuscript. All authors read and approved the final manuscript.

\section{Authors' information}

SS is the Head of the Division of Biochemical Diseases at British Columbia Children's Hospital $(\mathrm{BCCH})$ and Professor of Pediatrics at the Department of Pediatrics, University of British Columbia (UBC). SC and DL are Professors, KF 
is Director of the Biomedical Imaging Group, and DL is Postdoctoral Fellow in the Program in Molecular Medicine at the University of Massachusetts Medical School. EN is Postdoctoral Fellow and CS is PhD student at the Centre for Molecular Medicine and Therapeutics (CMMT). RS is a Pediatric Neurologist and Associate Professor in the Department of Pediatrics at the University Medical Center, Goettingen, Germany. CA is an Electrone Microscopist and Lecturer at the Hospital of Sickkids in Toronto, Ontario. NA is a Hemato-pathologist and Clinical Assistant Professor in the Department of Pathology and Laboratory Medicine, UBC. KS is a Pediatric Neurologist and Associate Clinical Professor in the Division of Pediatric Neurology, BCCH. MvA is a Clinical Geneticist and Clinical Professor in the Department of Medical Genetics, UBC. HV is the Director of the Biochemical Genetics and Provincial Newborn Screening Laboratory and Clinical Professor in the Department of Pathology, UBC. RW is Head of the Laboratory of Genetic Endocrine and Metabolic Diseases and Professor at Radboud University Nijmegen, The Netherlands. DR is a Pediatrician and Medical Geneticist at McGill University Health Centre and Director of the International Referral Centre for Vitamin B12 and Folate Disorders. DW is a Scientist in the Referral Centre for Vitamin B12 and Folate Disorders, at McGill University Health Centre. CJR is Associate Professor in the Department of Pediatrics, at UBC and CMMT. WW is Professor and EC is an Associate Professor in the Department of Medical Genetics, UBC, and CMMT. CvK is a Pediatrician and Biochemical Geneticist in the Division of Biochemical Diseases, BCCH, Assistant Professor at the Department of Pediatrics, CMMT/UBC, and Scholar of the Michael Smith Foundation for Health Research.

\section{Acknowledgements}

We gratefully acknowledge the family for their participation in this study; Dr Ebba Nexo, Arhus University Hospital, DK for transcobalamin measurements; Dr. S. Mercimek-Mahmutoglu for contributions in clinical care of this patient; Mrs. M. Higginson and Ms. M. Zhou for DNA extraction, sample handling and technical data; Dr. L. Zhang and Mrs. A. Tilups for preparation of fibroblasts for electron microscopy; Ms. M. Thomas and M. Lafek for consenting and data management.. This study is part of the Treatable Intellectual Disability Endeavour in British Columbia" [www.tidebc. org], supported by funding from the B.C. Children's Hospital Foundation as " $1^{\text {st }}$ Collaborative Area of Innovation"; the British Columbia Clinical Genomics Network [grant number BCCGN00031]; the Rare Diseases Foundation [grant number 2013-X] to SS:; Genome B.C. SOF5-021-R33 grant to CVK, SS, WW; the British Columbia Clinical Genomics Network (grant number BCCGN00031) to CVK; Canadian Institutes of Health Research \#301221 grant to CVK; and NIH PO1 DK60564 to SC. The authors have no conflict of interest to declare.

\section{Author details}

'Division of Biochemical Diseases, B.C. Children's Hospital, Vancouver, Canada. ${ }^{2}$ Department of Pediatrics, University of British Columbia, Vancouver, Canada. ${ }^{3}$ Treatable Intellectual Disability Endeavour in British Columbia, B.C. Children's Hospital, Vancouver, Canada. ${ }^{4}$ Center for Molecular Medicine and Therapeutics, Child and Family Research Institute, Vancouver, Canada. ${ }^{5}$ Department of Medical Genetics, University of British Columbia, Vancouver, Canada. ${ }^{6}$ Department of Pathology \& Laboratory Medicine, University of British Columbia, Vancouver, Canada. ${ }^{7}$ Division of Pediatric Neurology, B.C. Children's Hospital, Vancouver, Canada. ${ }^{8}$ Biochemical Genetics Laboratory, Children's and Women's Hospital, Vancouver, Canada. ${ }^{9}$ Program in Molecular Medicine, University of Massachusetts Medical School, Worcester, MA, USA.

${ }^{10}$ Biomedical Imaging Group, University of Massachusetts Medical School, Worcester, MA, USA. ${ }^{11}$ Department of Pediatrics, University Medical Center Goettingen, Goettingen, Germany. ${ }^{12}$ Division of Pathology, Hospital for Sick Children, Toronto, Ontario, Canada. ${ }^{13}$ Department of Laboratory Medicine, Radboud University Medical Centre, Nijmegen, The Netherlands. ${ }^{14}$ Department of Human Genetics, McGill University, Montreal, Québec, Canada.

Received: 20 April 2014 Accepted: 25 August 2014 Published online: 20 September 2014

\section{References}

1. van Karnebeek CD, Shevell M, Zschocke J, Moeschler JB, Stockler S: The metabolic evaluation of the child with an intellectual developmental disorder: Diagnostic algorithm for identification of treatable causes and new digital resource. Mol Genet Metab 2014, 111:428-438.
2. Nielsen E, Christoforidis S, Uttenweiler-Joseph S, Miaczynska M, Dewitte F, Wilm M, Hoflack B, Zerial M: Rabenosyn-5, a novel Rab5 effector, is complexed with hVPS45 and recruited to endosomes through a FYVE finger domain. J Cell Biol 2000, 151:601-612.

3. Morrison HA, Dionne H, Rusten TE, Brech A, Fisher WW, Pfeiffer BD, Celniker SE, Stenmark H, Bilder D: Regulation of early endosomal entry by the Drosophila tumor suppressors Rabenosyn and Vps45. Mol Biol Cell 2008, 19:4167-4176.

4. Mottola G, Classen AK, Gonzalez-Gaitan M, Eaton S, Zerial M: A novel function for the Rab5 effector Rabenosyn-5 in planar cell polarity. Development 2010, 137:2353-2364.

5. Navaroli DM, Bellve KD, Standley C, Lifshitz LM, Cardia J, Lambright D, Leonard D, Fogarty KE, Corvera S: Rabenosyn-5 defines the fate of the transferrin receptor following clathrin-mediated endocytosis. Proc Nat Acad Sci U S A 2012, 109:E471-E480.

6. Rahajeng J, Caplan S, Naslavsky N: Common and distinct roles for the binding partners Rabenosyn-5 and Vps45 in the regulation of endocytic trafficking in mammalian cells. Exp Cell Res 2010, 316:859-874.

7. Naslavsky N, Boehm M, Backlund PS Jr, Caplan S: Rabenosyn-5 and EHD1 interact and sequentially regulate protein recycling to the plasma membrane. Mol Biol Cell 2004, 15:2410-2422.

8. Eathiraj S, Pan X, Ritacco C, Lambright DG: Structural basis of family-wide Rab GTPase recognition by rabenosyn-5. Nature 2005, 436:415-419.

9. Hayakawa A, Hayes S, Leonard D, Lambright D, Corvera S: Evolutionarily conserved structural and functional roles of the FYVE domain. Biochem Soc Symp 2007, 74:95-105.

10. Mishra A, Eathiraj S, Corvera S, Lambright DG: Structural basis for Rab GTPase recognition and endosome tethering by the $\mathrm{C} 2 \mathrm{H} 2$ zinc finger of Early Endosomal Autoantigen 1 (EEA1). Proc Natl Acad Sci U S A 2010, 107:10866-10871.

11. Shaner NC, Lin MZ, McKeown MR, Steinbach PA, Hazelwood KL, Davidson MW, Tsien RY: Improving the photostability of bright monomeric orange and red fluorescent proteins. Nat Methods 2008, 5:545-551.

12. Steinfeld R, Reinhardt K, Schreiber K, Hillebrand M, Kraetzner R, Bruck W, Saftig P, Gartner J: Cathepsin D deficiency is associated with a human neurodegenerative disorder. Am J Hum Genet 2006, 78:988-998.

13. Bellve KD, Leonard D, Standley C, Lifshitz LM, Tuft RA, Hayakawa A, Corvera S, Fogarty KE: Plasma membrane domains specialized for clathrin-mediated endocytosis in primary cells. J Bio/ Chem 2006, 281:16139-16146.

14. Merrifield CJ, Perrais D, Zenisek D: Coupling between clathrin-coated-pit invagination, cortactin recruitment, and membrane scission observed in live cells. Cell 2005, 121:593-606.

15. Bonifacino JS, Traub LM: Signals for sorting of transmembrane proteins to endosomes and lysosomes. Annu Rev Biochem 2003, 72:395-447.

16. Dunn KW, McGraw TE, Maxfield FR: Iterative fractionation of recycling receptors from lysosomally destined ligands in an early sorting endosome. J Cell Biol 1989, 109:3303-3314.

17. Naslavsky N, McKenzie J, Altan-Bonnet N, Sheff D, Caplan S: EHD3 regulates early-endosome-to-Golgi transport and preserves Golgi morphology. J Cell Sci 2009, 122:389-400.

18. Siintola E, Partanen S, Stromme P, Haapanen A, Haltia M, Maehlen J, Lehesjoki AE, Tyynela J: Cathepsin D deficiency underlies congenital human neuronal ceroid-lipofuscinosis. Brain 2006, 129:1438-1445.

19. Varki A, Reitman ML, Vannier A, Kornfeld S, Grubb JH, Sly WS: Demonstration of the heterozygous state for I-cell disease and pseudo-Hurler polydystrophy by assay of $\mathrm{N}$-acetylglucosaminylphosphotransferase in white blood cells and fibroblasts. Am J Hum Genet 1982, 34:717-729.

20. Kudo M, Brem MS, Canfield WM: Mucolipidosis II (I-cell disease) and mucolipidosis IIIA (classical pseudo-hurler polydystrophy) are caused by mutations in the GlcNAc-phosphotransferase alpha / beta -subunits precursor gene. Am J Hum Genet 2006, 78:451-463.

21. Gliemann J: Receptors of the low density lipoprotein (LDL) receptor family in man: Multiple functions of the large family members via interaction with complex ligands. Biol Chem 1998, 379:951-964.

22. Stepensky $P$, Saada A, Cowan M, Tabib A, Fischer U, Berkun Y, Saleh H, Simanovsky N, Kogot-Levin A, Weintraub M, Ganaiem H, Shaag A, Zenvirt S, Borkhardt A, Elpeleg O, Bryant NJ, Mevorach D: The Thr224Asn mutation in the VPS45 gene is associated with the congenital neutropenia and primary myelofibrosis of infancy. Blood 2013, 121:5078-5087.

23. Vilboux T, Lev A, Malicdan MC, Simon AJ, Jarvinen P, Racek T, Puchalka J, Sood R, Carrington B, Bishop K, Mullikin J, Huizing M, Garty BZ, Eyal E, 
Wolach B, Gavrieli R, Toren A, Soudack M, Atawneh OM, Babushkin T, Schiby G, Cullinane A, Avivi C, Polak-Charcon S, Barshack I, Amariglio N, Rechavi G, van der Werff ten Bosch J, Anikster Y, Klein C, Gahl WA, Somech R: A congenital neutrophil defect syndrome associated with mutations in VPS45. N Engl J Med 2013, 369:54-65.

24. Tanaka T, Nakamura A: Oskar-induced endocytic activation and actin remodeling for anchorage of the Drosophila germ plasm. Bioarchitecture 2011, 1:122-126.

25. Blair RE, Sombati S, Lawrence DC, McCay BD, DeLorenzo RJ: Epileptogenesis causes acute and chronic increases in GABAA receptor endocytosis that contributes to the induction and maintenance of seizures in the hippocampal culture model of acquired epilepsy. J Pharmacol Exp Ther 2004, 310:871-880.

26. Boumil RM, Letts VA, Roberts MC, Lenz C, Mahaffey CL, Zhang ZW, Moser T, Frankel WN: A missense mutation in a highly conserved alternate exon of dynamin-1 causes epilepsy in fitful mice. PLoS Genet 2010, 6(8):e1001046.

27. Upreti C, Otero R, Partida C, Skinner F, Thakker R, Pacheco LF, Zhou ZY, Maglakelidze G, Veliskova J, Velisek L, Romanovicz D, Jones T, Stanton PK, Garrido-Sanabria ER: Altered neurotransmitter release, vesicle recycling and presynaptic structure in the pilocarpine model of temporal lobe epilepsy. Brain 2012, 135:869-885.

28. Gardiner J, Marc J: Disruption of normal cytoskeletal dynamics may play a key role in the pathogenesis of epilepsy. Neuroscientist 2010, 16:28-39.

29. Falace A, Filipello F, La Padula V, Vanni N, Madia F, De Pietri TD, de Falco FA Striano P, Dagna Bricarelli F, Minetti C, Benfenati F, Fassio A, Zara F: TBC1D24, an ARF6-interacting protein, is mutated in familial infantile myoclonic epilepsy. Am J Hum Genet 2010, 87:365-370.

doi:10.1186/s13023-014-0141-5

Cite this article as: Stockler et al: Single point mutation in Rabenosyn-5 in a female with intractable seizures and evidence of defective endocytotic trafficking. Orphanet Journal of Rare Diseases 2014 9:141.

\section{Submit your next manuscript to BioMed Central and take full advantage of:}

- Convenient online submission

- Thorough peer review

- No space constraints or color figure charges

- Immediate publication on acceptance

- Inclusion in PubMed, CAS, Scopus and Google Scholar

- Research which is freely available for redistribution 